\title{
LEVANTAMENTO FLORÍSTICO E CARACTERÍSTICAS DAS ESPÉCIES EM PRAÇAS PÚBLICAS EM LAGES-SC
}

\author{
FLORISTIC SURVEY AND CHARACTERISTICS OF SPECIES IN PUBLIC SQUARES \\ OF LAGES-SC
}

\author{
Fernanda Espíndola Assumpção Bastos ${ }^{1}$; Samila Silva Camargo; Aline Meneguzzi²; \\ Aike Anneliese Kretzschmar ${ }^{3}$; Leo Rufato ${ }^{3}$
}

\section{RESUMO}

Na composição florística de praças e jardins é importante que haja diversidade de famílias e espécies, o que atrai uma fauna diversificada e a ornamentação torna-se rica. O objetivo desse trabalho foi avaliar a composição florística e caracterizar as espécies com relação a toxidade, presença de espinhos, propriedades medicinais e alimentícias. O levantamento florístico foi realizado nas praças públicas João Ribeiro, Vidal Ramos Sênior e Joca Neves, localizadas no município de Lages, Santa Catarina, realizado em junho a dezembro de 2013. As espécies foram identificadas pelo nome popular, nome científico e família botânica e todas as plantas foram fotografadas para facilitar as identificações. Foram registradas 50 espécies, distribuídas em 34 famílias. As famílias com maior ocorrência foram Fabaceae e Poaceae, cada qual com cinco espécies, representando $19,2 \%$ do total de espécies. Em segundo lugar a família Myrtaceae com quatro espécies (7,7\%), seguida pelas famílias Cupressaceae, Iridaceae, Arecaceae e Lythraceae, com duas espécies cada (15,2\%). O restante das famílias foi representado por uma espécie em cada (57,9\%). A Araucaria angustifolia (Pinheiro-brasileiro) foi a espécie de maior frequência encontrada nos três locais de estudo, seguido pelo Tagetes erecta (Tagetes), Ophiopogon intermedius (Grama-preta), Ligustrum lucidum (Ligustro) e Lagerstroemia indica (Resedá), estes encontrados em dois dos locais inventariados. Identificou-se uma grande variedade de famílias botânicas nos locais de estudo, ainda que com poucas espécies representando cada uma das famílias. O conhecimento da diversidade de famílias botânicas em espaços públicos pode trazer contribuições tanto de manejo quanto em termos culturais, históricos, e inclusive de saúde pública.

Palavras-chave: Espaços urbanos; Paisagismo; Serra Catarinense; Biodiversidade.

\begin{abstract}
In the floristic composition of squares and gardens is important to have diversity of families and species, which attracts a diverse fauna and ornamentation becomes rich. The aim of this study was to evaluate the floristic composition and characterize the species with respect to toxicity, the presence of thorns, medicinal and food properties. The floristic survey was carried out in public the squares João Ribeiro, Vidal Ramos Sênior. and Joca Neves, located in Lages, Santa Catarina, held in June and December of 2013. The plant species were identified by common name, scientific name and botanical family and all plants were photographed to facilitate identification. There were recorded 50 species belonging to 34 families. Families with higher incidence were Fabaceae and Poaceae, with five species each, representing $19.2 \%$ of the total species. Secondly, the Myrtaceae family with four species (7.7\%), followed by Cupressaceae, Iridaceae, Arecaceae and Lythraceae, with two species each (15.2\%). The remaining families were represented by one species each (57.9\%). The Araucaria angustifolia (Pinheiro of Brazil) was the species most often found in the three study sites, followed by Tagetes erecta (Tagetes), Ophiopogon intermedius (Black-grass), Ligustrum lucidum (Privet) and Lagerstroemia indica (Mignonette), these found in two of the inventory locations. There were identified a wide variety of plant families in the study sites, although with a few species representing each family. Knowledge of the diversity of plant families in public spaces can bring contributions for management as well in cultural, historical, and even public health.
\end{abstract}

Keywords: Urban spaces; Landscaping; Serra Catarinense; Biodiversity.

Recebido em 09.03.2016 e aceito em 01.06.2016

1 Engenheira Agrônoma, MSc., Doutoranda em Produção Vegetal pela Universidade do Estado de Santa Catarina, Lages - Santa Catarina. Email: feabastos@gmail.com; samilasc@yahoo.com.br

2 Engenheira Florestal, Mestranda em Engenharia Florestal pela Universidade do Estado de Santa Catarina, Lages - Santa Catarina. Email: alinemeneguzzi@yahoo.com.br

3 Engenheiro Agrônomo, Dr., Professor do Departamento de Agronomia Universidade do Estado de Santa Catarina, Lages - Santa Catarina. Email: aikeanneliese@yahoo.com.br; leoruffato@yahoo.com.br 


\section{INTRODUÇÃO}

As cidades representam os pontos mais expressivos de mudança de natureza física, devido a ação do homem, e isso acarreta em modificações da paisagem natural, devido a dinamicidade antropogenética ligada aos sistemas políticos e econômicos dominantes ao longo da história (SILVEIRA; BARROS, 2004). No entanto, nas cidades existem espaços públicos como as praças, usadas como referencial urbano marcado pela convivência humana, servindo como um importante equipamento histórico cultural que, especialmente no Brasil, expressa o surgimento e desenvolvimento de inúmeras cidades (ROMANI et al., 2012). Áreas verdes são tidos como espaços livres onde predominam áreas plantadas de vegetação, correspondendo, em geral, ao que se conhece como parques, jardins ou praças (LLARDENT, 1982). Entre outras funções, a arborização urbana também cumpre a função ecológica de fornecer abrigo e alimentação à fauna autóctone, viabilizando em parte a vida silvestre no interior das cidades (DANTAS; SOUZA, 2004). Além disso é fato que as árvores melhoram a qualidade do ar e do solo, fornecem abrigo e alimentos aos animais, funcionam como barreiras acústicas, valorizam os imóveis e contribuem para a beleza natural da cidade e seus valores culturais (MACIEL et al., 2008).

Diagnosticar essas áreas é importante para a elaboração do planejamento da arborização de uma cidade (SCHWAB et al., 2014), pois além da elevada área física, a cobertura vegetal é uma característica marcante nestes espaços, sendo um dos componentes de grande importância na formação da paisagem local (SILVA; MEUNIER; MIRANDA, 2007). Para conhecer a arborização da cidade, é necessário fazer um levantamento florístico para avaliar a situação e, por meio dos resultados obtidos, será possível verificar as soluções possíveis para cada local (ASSUNÇÃO et al., 2014).

Segundo Dias (1996) é necessário conhecer as características das espécies utilizadas na arborização como o porte e o tamanho, período e características de frutificação, forma do tronco, toxicidade, presença de espinhos, emissão de odores, agressividade das raízes, velocidade de crescimento, durabilidade, rusticidade, resistência à poluição, impacto de pedestres, pragas e doenças, resistência ao vento, à seca e outros.

As cidades brasileiras enfrentam sérios problemas acarretados pelo mau planejamento da arborização urbana, os quais podem causar danos materiais e humanos. Os principais são relacionados ao uso inadequado de espécies arbóreas, e pela queda de árvores de grande porte, (MOSER et al., 2010), ou uso de plantas tóxicas e alergênicas (KÜSTER et al., 2012).

Dessa forma, objetivou-se com esse estudo realizar um levantamento florístico das plantas localizadas em três praças distintas na cidade de Lages (SC), a fim de caracteriza-las em quanto a sua origem, e o risco que elas representam por toxicidade e presença de espinhos 
para aportar informações que amparem o planejamento da arborização e do paisagismo existente nestes locais.

\section{MATERIAL E MÉTODOS}

O levantamento florístico foi realizado na cidade de Lages, Santa Catarina, Brasil. O município possui $2.631,504$ km² de área territorial e uma população de 158.846 habitantes, cujo marco zero localiza-se a uma latitude de 27ㅇ 48' 58" Sul, a uma longitude de 50 19' 34" Oeste e altitude de 940 metros (IBGE, 2010). Segundo classificação de Köppen o clima é caracterizado por invernos e verões amenos (Cfb).

As informações das plantas inventariadas foram observadas em três praças públicas: João Ribeiro, Vidal Ramos Sênior e Joca Neves, inseridas na região central da cidade, e escolhidas ao acaso, levando-se em conta apenas a informação de que são utilizadas diariamente por diversos cidadãos

As plantas foram identificadas pelo nome popular, nome científico e família botânica e todas as plantas foram fotografadas para facilitar as identificações.

O estudo foi realizado nos meses de junho a dezembro de 2013. Para cada espécie encontrada nas praças, foram obtidas, em literatura, informações a respeito da origem, presença de substâncias tóxicas, espinhos e se são plantas úteis, ou seja, se possuem características alimentícias ou medicinais para os usuários das praças.

Foi estimada a frequência relativa de cada espécie, calculada pela razão entre o número de indivíduos da espécie e o número total de indivíduos dentro de cada grupo multiplicada por 100, de acordo com metodologia utilizada por Schwab et al. (2014).

\section{RESULTADOS E DISCUSSÃO}

No total, foram registradas 50 espécies, distribuídas em 34 famílias (Figura 1).

As famílias encontradas foram Fabaceae e Poaceae, com cinco espécies cada, ou seja, 9,6\% das famílias encontradas. Em levantamento realizado por De Freitas, Pinheiro e Abrahão (2015), em quatro praças na zona norte da cidade do Rio de Janeiro, RJ, a análise quantitativa assemelhou-se ao presente estudo, demonstrando que a família botânica representada pelo maior número de espécies foi Fabaceae, com 13 espécies. No Brasil, com relação a esta família, ocorrem cerca de 220 gêneros, 2807 espécies, e 729 variedades, ocorrendo em todo território nacional, com hábitos diversos, encontrando-se desde árvores e arbustos até ervas (LIMA et al., 2015) 


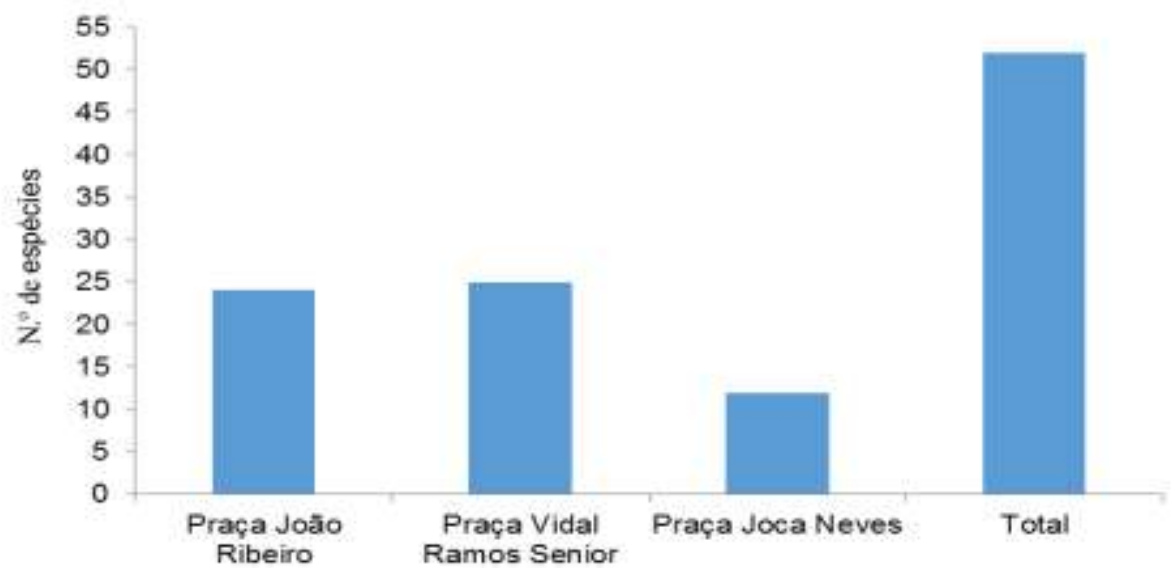

Figura 1. Número de espécies de plantas ornamentais nas praças públicas João Ribeiro, Vidal Ramos Sênior e Joca Neves, Lages - SC

Figure 1. Number of ornamental plant species in the public squares João Ribeiro, Vidal Ramos Sênior and Joca Neves, Lages - SC

Na sequência, as famílias que aparecem com maior frequência são Myrtaceae (7,7\%) com quatro espécies, Cupressaceae, Iridaceae, Arecaceae e Lythraceae $(3,8 \%)$ com duas espécies cada, e as famílias Araucariaceae, Oleaceae, Rosaceae, Ruscaceae e Anthericaceae com uma espécie cada (1,9\%).

Com relação a existência nos locais de plantas tóxicas, com espinhos e úteis, constatou-se que nas três praças há presença de plantas com espinhos ou superfícies pontiagudas e espécies para fins medicinais ou culinários. Na Praça João Ribeiro, das 24 espécies existentes, cinco oferecem risco por toxicidade ou por presença de espinhos (Figura 2), já as espécies Tagetes erecta e Hedychium chrysoleucum (Tabela 1) apresentam propriedades medicinais.

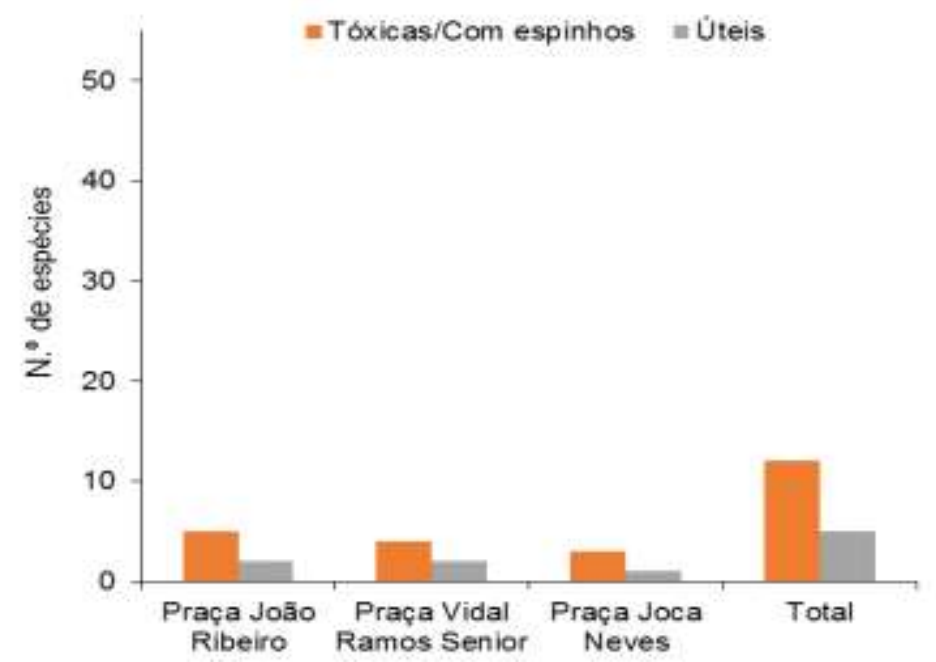

Figura 2. Número de espécies de plantas ornamentais tóxicas e com espinhos e número de plantas úteis nas praças públicas João Ribeiro, Vidal Ramos Sênior e Joca Neves, em Lages - SC

Figure 2. Number of toxic ornamental plant species and with thorns and number of useful plants in the public squares João Ribeiro, Vidal Ramos Sênior and Joca Neves in Lages - SC 
Tabela 1. Lista de espécies de plantas ornamentais inventariadas na Praça João Ribeiro Table 1. List of ornamental plant species inventoried in João Ribeiro Square

\begin{tabular}{|c|c|c|c|}
\hline Nomes Populares & Nomes Científicos & Família Botânica & Origem \\
\hline Araucária & Araucaria angustifolia (Bertol.) Kuntze & Araucariaceae & Nativa \\
\hline Ardísia & Ardisia crenata Sims & Myrcinaceae & Exótica \\
\hline Azaléia-branca & Rhododendron simsii Planch. & Ericaceae & Exótica \\
\hline Azaléia-rosa & Rhododendron simsii Planch. & Ericaceae & Exótica \\
\hline Buxinho & Buxus sempervirens Linn & Buxaceae & Exótica \\
\hline Capim-do-texas & Pennisetum setaceum (Forsk.) Chiov & Poaceae & Exótica \\
\hline Cica & Cycas revoluta Thunb. & Cycadaceae & Exótica \\
\hline Cipreste & Chamaecyparis obtusa Sieb. \& Zucc. & Cupressaceae & Exótica \\
\hline Clorofito & Chlorophytum comosum Baker & Agavaceae & Exótica \\
\hline Estrelitzia & Strelitzia reginae Banks & Strelitziaceae & Exótica \\
\hline Flamboyant & Delonix regia (Bojer ex Hook.) Raf. & Fabaceae & Exótica \\
\hline Fórmium & Phormium tenax Forst. & Hemerocallidaceae & Exótica \\
\hline Grama-esmeralda & Zoysia japonica Steud. & Poaceae & Exótica \\
\hline Grama-preta & Ophiopogon intermedius D. Don & Ruscaceae & Exótica \\
\hline Hortensia & Hydrangea macrophylla Ser. & Hydrangeaceae & Exótica \\
\hline Íris & Iris germanica Linn & Iridaceae & Exótica \\
\hline Ligustro & Ligustrum sinense Lour. & Oleaceae & Exótica \\
\hline Lírio-amarelo-do-brejo & Hedychium chrysoleucum Koehne & Zingiberaceae & Exótica \\
\hline Moréia & Dietes bicolor Sweet ex G.Don & Iridaceae & Exótica \\
\hline Pitosporum & Pittosporum tobira (Thunb.) Ait. & Pittosporaceae & Exótica \\
\hline Quaresmeira & Tibouchina granulosa Cogn. & Melastomataceae & Nativa \\
\hline Resedá & Lagerstroemia indica Linn & Lythraceae & Exótica \\
\hline Tagetes & Tagetes erecta Linn & Asteraceae & Exótica \\
\hline Tuia & Cupressus macrocarpa Hartw. & Cupressaceae & Exótica \\
\hline
\end{tabular}

Na Praça Vidal Ramos Sênior, das 25 espécies levantadas, três possuem espinhos ou folhas pontiagudas e Schinus molle (aroeira-salsa), apresenta toxidez. Nesta mesma praça, Psidium cattleyanum (araçá) produz frutos comestíveis e Rosmarinus officinalis (alecrim) (Tabela 2) é uma erva amplamente utilizada na medicina popular em cosméticos, fitofármacos e também para dar aroma aos alimentos (BOZIN et al., 2007). Küster et al.(2012) verificaram em outras áreas de Lages-SC, que do total de indivíduos amostrados, 51,52\% das espécies são consideradas como tóxicas, alergênicas ou apresentam algum tipo de risco às pessoas. De acordo com Vasconcelos, Vieira e Vieira (2009), plantas tóxicas são assim denominadas por apresentarem substâncias biodisponíveis capazes de causar alterações metabólicas, que são reconhecidas como sintomas de intoxicação, que em alguns casos podem causar sérios transtornos e até mesmo levar a óbito. No Brasil, a cada dez casos de intoxicação por plantas, seis ocorrem em crianças menores de nove anos, devido à presença comum em ambientes públicos. Nas três praças estudadas em Lages-SC não há qualquer identificação ou alerta sobre as plantas tóxicas. Para Vasconcelos; Vieira e Vieira (2009), uma possível solução para a prevenção de acidentes, seria a identificação das espécies com placas informando sobre os 
riscos, além de atividades educativas que informem as crianças sobre os riscos de brincarem ou colocarem plantas na boca.

Tabela 2. Lista de espécies de plantas ornamentais inventariadas na Praça Vidal Ramos Sênior Table 2. List of ornamental plant species inventoried in Vidal Ramos Sênior Square

\begin{tabular}{|c|c|c|c|}
\hline Nome Popular & Nome Científico & Família Botânica & Origem \\
\hline Alecrim & Rosmarinus officinalis Linn & Lamiaceae & Exótica \\
\hline Araçá & Psidium cattleyanum Sabine & Myrtaceae & Nativa \\
\hline Araucária & Araucaria angustifolia (Bertol.) Kuntze & Araucariaceae & Nativa \\
\hline Aroeira-salsa & Schinus molle L. & Anacardiacea & Nativa \\
\hline Bromélia-de-sol & Aechmea blanchetiana (Baker) L.B.Sm. & Bromeliaceae & Nativa \\
\hline Buganvilha-branca & Bougainvillea glabra Choisy & Nyctaginaceae & Nativa \\
\hline Capim-chorão & Eragrostis curvula (Schrader) Nees & Poaceae & Exótica \\
\hline Capim-do-Texas & Pennisetum setaceum (Forsk.) Chiov & Poaceae & Exótica \\
\hline Clorofito & Chlorophytum comosum Baker & Agavaceae & Exótica \\
\hline Coqueiro-do-campo & Syagrus flexuosa (Mart.) Beccari & Arecaceae & Nativa \\
\hline Cortaderia & $\begin{array}{l}\text { Cortaderia selloana (Schult. \& Schult. f.) } \\
\text { Asch. \& Graebn. }\end{array}$ & $\mathrm{Pc}$ & ativa \\
\hline Érica & Cuphea gracilis Kunth & Lythraceae & Nativa \\
\hline Escova-de-garrafa & Callistemon spp & Myrtaceae & Exótica \\
\hline Eucalipto & Eucalyptus globulus L. & Myrtaceae & Exótica \\
\hline Flor-de-coral & Russelia equisetiformis Schltdl. \& Cham. & Plantaginaceae & Exótica \\
\hline Grama-preta & Ophiopogon intermedius D. Don & Ruscaceae & Exótica \\
\hline Hemerocallis-amarelo & Hemerocallis flava Linn & Hemerocallidaceae & Exótica \\
\hline Hemerocallis-rosa & Hemerocallis $x$ hybrida Hort & Hemerocallidaceae & Exótica \\
\hline Lírio-Laranja & Lilium pumilum DC. & Liliaceae & Exótica \\
\hline Palmeira-Phoênix & Phoenix roebelenii O'Brien & Arecaceae & Exótica \\
\hline Plátano & Platanus $x$ hispanica Miller ex Münchh & Platanaceae & Exótica \\
\hline Podocarpus & Podocarpus macrophyllus (Thunb.) Sweet & Podocarpaceae & Exótica \\
\hline Resedá & Lagerstroemia indica Linn & Lythraceae & Exótica \\
\hline Tagetes & Tagetes erecta Linn & Asteraceae & Exótica \\
\hline Tipuana & Tipuana tipu (Benth.) & Fabaceae & Exótica \\
\hline
\end{tabular}

Na Praça Joca Neves foram inventariadas 11 espécies, das quais Nerium oleander, Ligustrum lucidum e Melia azedarach (Tabela 3) apresentam toxidez e Feijoa sellowiana (goiaba-serrana) (Tabela 3) possui frutos comestíveis.

Coletto, Muller e Wolski (2008) afirmam que o uso de plantas frutíferas nativas na arborização, é recomendado sob o ponto de vista ambiental, para propiciar a atração da fauna silvestre para a cidade. O restante das famílias foi representado por uma espécie em cada e totalizaram 57,9\% das espécies. Ligustrum lucidum é uma espécie nativa da Ásia, considerada pelo Instituto Hórus (2011) como invasora biológica no sul do Brasil, e a alta preferência por essa espécie contribui para a baixa diversidade na arborização. A Araucária (Araucaria angustifolia) foi encontrada nos três locais de estudo. Tagetes (Tagetes erecta), Grama-preta 
(Ophiopogon intermedius), Ligustro (Ligustrum lucidum) e Resedá (Lagerstroemia indica) foram verificados em dois dos locais inventariados.

Tabela 3. Lista de espécies de plantas ornamentais inventariadas na Praça Joca Neves Table 3. List of ornamental plant species inventoried in Joca Neves Square

\begin{tabular}{llll}
\hline Nome Popular & Nome Científico & Família Botânica & Origem \\
\hline Angico & Parapiptadenia rigida (Benth.) Brenan. & Fabaceae & Nativa \\
Araucária & Araucaria angustifolia (Bertol.) Kuntze & Araucariaceae & Nativa \\
Ardísia & Ardisia crenata Sims & Myrcinaceae & Exótica \\
Chorão-verdadeiro & Salix babylonica L. & Salicaceae & Exótica \\
Cinamomo & Melia azedarach L. & Meliaceae & Exótica \\
Espirradeira & Nerium oleander L. & Apocynaceae & Exótica \\
Figueira-do-mato & Ficus insipida Willd. & Moraceae & Exótca \\
Goiabeira-serrana & Feijoa sellowiana (O.Berg) Burret & Myrtaceae & Nativa \\
Ipê-amarelo & Tabebuia chrysotricha (Mart. Ex dc.) Sandl. & Fabaceae & Nativa \\
Ipê-roxo & Tabebuia impetiginosa Martius ex A. P. de Candolle & Fabaceae & Nativa \\
Ligustro & Ligustrum sinense Lour. & Oleaceae & Exótica \\
\hline
\end{tabular}

Com relação a origem das 50 espécies levantadas, ainda nas tabelas 1, 2 e 3, apenas 13 delas (25\%) são nativas do Brasil, sendo que as outras 39 espécies (75\%) são espécies exóticas. O baixo número de espécies nativas nos locais de estudo não é benéfico, pois segundo Heiden, Barbieri e Stumpf (2006), o uso de espécies exóticas no paisagismo contribui para a uniformização das paisagens, enquanto o uso de espécies nativas, ao mesmo tempo em que ajuda na preservação da flora local, proporciona o reforço das identidades regionais. Devido à grande diversidade de funções que esta vegetação desempenha nos ambientes urbanos, fazem-se necessários critérios rígidos na seleção de espécies, tanto com relação ao ecossistema local, como em relação ao público que convive nestes espaços (BIONDI e LEAL, 2008), portanto faz-se necessário um melhor planejamento paisagístico nos locais de estudo, focando no maior uso de plantas nativas ornamentais.

\section{CONCLUSÕES}

Identificou-se uma variedade de famílias botânicas nos locais de estudo, ainda que com poucas espécies representando cada uma delas.

Foram registradas 50 espécies de plantas no levantamento, distribuídas em 34 famílias, com maior ocorrência de Fabaceae e Poaceae, com 19,2\%; Constatou-se a ocorrência de espécies que oferecem risco aos frequentadores das praças pela toxicidade ou presença de espinhos.

Do total de espécies levantadas nas três praças, somente $26 \%$ são nativas, sendo os 74\% restantes de espécies exóticas do Brasil. 


\section{AGRADECIMENTOS}

À CAPES (Coordenação de Aperfeiçoamento de Pessoal de Nível Superior), FAPESC (Fundação de Amparo à Pesquisa em Santa Catarina) e à UDESC (Universidade do Estado de Santa Catarina) pelas bolsas de estudos e financiamento dessa pesquisa.

\section{REFERÊNCIAS}

ASSUNÇÃO, K. C. de; LUZ, P. B. da; NEVES, L. G.; SOBRINHO, S. de P. Levantamento quantitativo da arborização de praças da cidade de Cáceres/MT. Revista da Sociedade Brasileira de Arborização Urbana, Piracicaba, v. 9, n. 1, p.123-132, 2014.

BIONDI, DANIELA; LEAL, LUCIANA. Caracterização das plantas produzidas no Horto Municipal da Barreirinha-Curitiba/PR. Revista da Sociedade Brasileira de Arborização Urbana, Piracicaba, v. 3, n. 2, p. 20-36, 2008.

BOZIN, B., MIMICA-DUKIC, N., SAMOJLIK, I.; JOVIN, E. Antimicrobial and antioxidant properties of rosemary and sage (Rosmarinus officinalis L. and Salvia officinalis L., Lamiaceae) essential oils. Journal of agricultural and food chemistry, Washington, DC, v. 55, n. 19, p. 7879-7885, 2007.

COLETTO, E. P.; MULLER, N. G.; WOLSKI, S. S. Diagnóstico da Arborização das Vias Públicas do Município de Sete de Setembro - RS. Revista da Sociedade Brasileira de Arborização Urbana, Curitiba, v. 3, n. 2, p.110-122, 2008.

DANTAS, I. C.; SOUZA, C. M. C. Arborização urbana na cidade de Campina Grande - PB: inventário e suas espécies. Revista de Biologia e Ciências da Terra, Campina Grande, v. 4, n. 2, 2004.

DIAS, R. A importância da arborização urbana. SEMINÁRIO DE ARBORIZAÇÃO URBANA NO RIO DE JANEIRO, v. 1, p. 29-33, 1996.

FREITAS, W. K.; PINHEIRO, M. A. S.; ABRAHÃO, L. L. F. Análise da Arborização de Quatro Praças no Bairro da Tijuca, RJ, Brasil. Floresta e Ambiente, Rio de Janeiro, v. 22, n. 1, p. 23$31,2015$.

HEIDEN, G.; BARBIERI, R. L.; STUMPF, E. R. T. Considerações sobre o uso de plantas ornamentais nativas. Revista Brasileira de Horticultura Ornamental, Campinas, v. 12, n. 1, p. 2-7, 2006.

IBGE. Censo Demográfico 2010. Agregado por Setores censitários dos resultados do universo. Rio de Janeiro: IBGE, 2010. Disponível em: www.ibge.gov.br/censo2010. Acesso: 30/06/15. Instituto Hórus. 2011. Base de dados de espécies exóticas invasoras no Brasil. www.institutohorus.org.br. Acessado em: 22 Fev 2011.

KÜSTER, L. C.; STEDILLE, L. I. B.; DACOREGIO, H.; SILVA, A. C.; HIGUCHI, P. Avaliação de riscos e procedências de espécies arbóreas nas escolas estaduais de Lages, SC. Revista de Ciências Agroveterinárias, Lages, v.11, n.2, p.118-125, 2012.

LIMA, H. C. DE; QUEIROZ, L. P.; MORIM, M. P.; SOUZA, V. C.; DUTRA, V. F.; BORTOLUZZI, R. L. C.; IGANCI, J. R. V.; FORTUNATO, R. H.; VAZ, A. M. S. F.; SOUZA, E. R. DE; FILARDI, F. L. R.; VALLS, J. F. M.; GARCIA, F. C. P.; FERNANDES, J. M.; MARTINS-DA-SILVA, R. C. 
V.; PEREZ, A. P. F.; MANSANO, V. F.; MIOTTO, S. T. S.; TOZZI, A. M. G. A.; MEIRELES, J. E.; LIMA, L. C. P. ; OLIVEIRA, M. L. A. A.; FLORES, A. S.; TORKE, B. M.; PINTO, R. B.; LEWIS, G. P.; BARROS, M. J. F.; SCHÜTZ, R.; PENNINGTON, T.; KLITGAARD, B. B.; RANDO, J. G.; SCALON, V. R.; CARDOSO, D. B. O. S.; COSTA, L. C. DA; SILVA, M. J. DA; MOURA, T. M.; BARROS, L. A. V. DE; SILVA, M. C. R.; QUEIROZ, R. T.; SARTORI, A. L. B.; CAMARGO, R. A.; LIMA, I. B.; COSTA, J.; SOARES, M. V. B.; SNAK, C.; SÃO-MATEUS, W.; FALCÃO, M. J.; MARTINS, M. V.; REIS, I. P.; CORDULA, E. Fabaceae in Lista de Espécies da Flora do Brasil. Jardim Botânico do Rio de Janeiro. Disponível em: <http://floradobrasil.jbrj.gov.br/jabot/floradobrasil/FB115>. Acesso em: 26 Out. 2015.

MACIEL, L.J.; COGNATO, B.B; BOFFIL, M.C.; SILVA, G. F.; BORTOLI,R.; MUHLE,P.R.; LUCHESE,S.; SILVA,S.R.K.; CARDONE,B.L.; SILVA,V.G.; GONÇALVES,B.P.R.; CORTEZ,R.S.L. Educação Ambiental como ferramenta para a manutenção da arborização urbana de Porto Alegre - RS. In: Congresso Brasileiro de Arborização Urbana; 2008, Manaus. Anais...Manaus: SBAU, 2008. Vol. 12, 5 p.

MOSER, P.; SILVA, A.C.; HIGUCHI, P.; SANTOS, E. M.; SCHMITZ, V. Avaliação póstempestade da arborização do campus da Universidade do Estado de Santa Catarina em Lages, SC. Revista da Sociedade Brasileira de Arborização Urbana, Piracicaba, v. 5, n.2 p.36-46, 2010.

ROMANI, G. N.; GIMENES, R.; SILVA, M. T.; PIVETTA. K. F. L.; BATISTA; G. S. Análise qualiquantitativa da arborização na praça XV de novembro em Ribeirão Preto - SP, Brasil. Revista Arvore, Viçosa, v. 36, n. 3, p. 479-487, 2012.

SCHWAB, N. T.; GIRARDI, L. B.; NEUHAUS, M.; BACKES, F. A. A. L.; BELLÉ, R. A.; MENEGAES, J. F. Diversidade florística do bairro Nossa Senhora das Dores em Santa Maria, RS. Revista Brasileira de Horticultura Ornamental, Campinas, v. 20, n. 2, p.155-162, 2014.

SILVA, L. R.; MEUNIER, I. M. J.; MIRANDA, A. M. F. Riqueza e densidade de árvores, arvoretas e palmeiras em parques urbanos de Recife, Pernambuco, Brasil. Revista da Sociedade Brasileira de Arborização Urbana, Curitiba, v. 2, n. 4, p. 34-49, 2007.

SILVEIRA, G.; BARROS, M. V. F. Perfil geoambiental de praças: região norte na cidade de Londrina-PR. Semina: Ciências Exatas e Tecnológicas, Londrina, v. 22, n. 1, p. 63-71, 2004.

VASCONCELOS, J.; VIEIRA, J. G. P.; VIEIRA, E. P. P. Plantas tóxicas: conhecer para prevenir. Revista Científica da UFPA, Belém, v. 7, n. 01, p. 1-6, 2009. 